\title{
ADMINISTRACIÓN DE RIESGOS MEDIANTE LA TEORÍA DE RIESGOS EXTREMOS
}

\author{
Pablo Pérez Akaki.*
}

Escuela de Graduados en Administración y Dirección de Empresas

Tecnológico de Monterrey, Campus Estado de México

(Recibido 24 de enero 2005, aceptado 10 de marzo 2005 )

\section{Resumen}

En este trabajo se ofrece una medición empírica del Valor en Riesgo (VaR) utilizando la teoría de eventos extremos (EVT), teoría originalmente desarrollada en el ámbito asegurador y que recientemente está siendo utilizada como una medida de administración de riesgos. Se presentan dos aproximaciones empíricas para demostrar que la EVT no solo puede usarse para eventos catastróficos, como es normalmente empleada, sino también en la administración de riesgos de un portafolio de inversiones y la medición de los riesgos de las colas de una cartera de seguros.

\section{Abstract}

This paper offers an empirical estimation of Value at Risk (VaR) using Extreme Value Theory (EVT), a theory originally developed in insurance, that recently has been widely used in financial risk management. This paper offers two cases that show that EVT can be used, additionally to catastrophic events in insurance, in risk management in a financial portfolio and to describe the tails in a insurance pool.

Clasificación JEL: C16, D81, G22

Palabras clave: VaR, EVT, Riesgos financieros, Carteras de seguros

* Carretera Lago de Guadalupe Km. 3.5, Col. Margarita Maza de Juárez, Atizapán de Zaragoza, c.p. 52926, Estado de México, México. Correo electrónico: ppablo@itesm.mx 


\section{Introducción}

En los últimos años, a partir de la gran volatilidad que exhiben las variables financieras, los modelos de administración de riesgos han ganado gran aceptación entre las empresas, particularmente aquellas en el sector financiero, pues ofrecen mecanismos de cobertura ante los eventos de riesgo que ponen en riesgo los resultados financieros, ya sea en las inversiones disminuyendo sus retornos o en los pasivos, aumentando su monto.

Dentro de esta corriente se encuentra la teoría del valor extremos (Extreme Value Theory, EVT), originalmente propuesta en el ámbito de los seguros para la administración de riegos catastróficos, pero que recientemente comienza a ser utilizada no solo en estos contextos, sino en otros más generales de análisis de riesgos financieros.

Es en esa línea donde el presente trabajo desea contribuir, pues tiene como objetivo el proponer a los modelos de riesgos extremos como medida de administración de riesgos, que así como el Valor en Riesgo, ofrece posibilidades de definir límites a la retención de riesgos por parte de los intermediarios financieros y, consecuentemente, define los riesgos a cubrir ya sea por otra institución o por el mercado. Este trabajo también tiene como objetivo aportar un par de casos de aplicación de la teoría de los eventos extremos en la administración de los riesgos financieros y operativos de una empresa de seguros, con la finalidad de demostrar que la teoría no solo corresponde a eventos catastróficos, sino que puede ser utilizada en riesgos tan comunes como los de los rendimientos de un portafolio de inversiones y las reclamaciones de una cartera de seguros.

Para conseguir este propósito, el trabajo se estructura de la siguiente manera: en la sección 2 ofrece los elementos teóricos de la teoría de eventos extremos, abordando el modelo de bloque máximo y el de picos sobre el umbral; en la sección 3 se discute sobre la administración de riesgos y se particulariza en el Valor en Riesgo, la medida que ha sido propuesta en los acuerdos de Basilea que sirve como referencia para los intermediarios financieros en la constitución de reservas contra riesgos "extremos"; la sección 4 utiliza el modelo de picos sobre el umbral para dos casos de análisis del mercado de seguros, el de la formación de un portafolio de inversiones restringido en los montos a invertir en los diferentes instrumentos que el mercado ofrece, restricción por regulación de las autoridades financieras para proteger los recursos de terceros, y el de la administración de una cartera de seguros de gastos médicos donde se presentan extremos que siguen el comportamiento propuesto por la teoría de eventos extremos. Finalmente se ofrecen algunas conclusiones del trabajo.

\section{La teoría de eventos extremos}

Las teorías de eventos extremos se han constituido como una herramienta de gran utilidad para describir de manera precisa las colas de una distribución de probabilidad, que es la parte de una función de probabilidad donde se identifican los eventos de poca frecuencia pero gran impacto económico, a lo que les ha dado el nombre de riesgos extremos.

Estas teorías parten del principio "Dejar que las colas hablen" en el cual se expresa que por si solas, las colas pueden presentar comportamientos diferentes al del resto de eventos de una población de estudio y dada su naturaleza poco 
frecuente y de gran impacto, es necesario su estudio particular para poder caracterizarlas de una mejor manera.

La teoría del valor extremo ofrece varias aproximaciones metodológicas para su estudio, pero aquí se van a presentar solamente las dos más importantes: por un lado el modelo de bloque máximo en donde se presenta la función generalizada del valor extremo y por el otro, se tiene el modelo de picos sobre el umbral (peaks over the threshold), que ofrece una aproximación al comportamiento de los eventos de gran magnitud, pero medidos como excedentes sobre una cota inferior. A continuación se presentan ambos métodos.

\subsection{Teoría clásica de eventos extremos: modelo de bloque máximo}

La teoría clásica de la distribución de eventos extremos parte de reconocer la existencia de una serie de variables aleatorias secuenciales $X_{1}, X_{2}, \cdots$, idénticamente distribuidas. El estadístico $M_{n}=\operatorname{Max}\left\{X_{1}, \cdots, X_{n}\right\}$ denota el $n$-ésimo máximo muestral del proceso. Suponga que existen $a_{n}>0$ y $b_{n}$, tal que

$$
\operatorname{Pr}\left\{\frac{M_{n}-b_{n}}{a_{n}} \leq x\right\}=F\left(a_{n} x+b_{n}\right)^{n} \rightarrow H(x),
$$

lo cual quiere decir que una transformación del estadístico $M_{n}$ por las constantes $a_{n}$ y $b_{n}$ tiende en probabilidad a una función de distribución $H(x)$ independientemente de la distribución que caracterice a $M_{n}$.

El teorema de tres tipos (Three Types Theorem) o teorema de Fisher-Tippet sostiene que si una función no degenerada $H$ existe, debe ser de alguno de los tres tipos siguientes:

$$
\begin{gathered}
H(x)=\exp \left(-e^{-x}\right) \forall x, \\
H(x)= \begin{cases}0, & x<0 \\
\exp \left(-x^{-\alpha}\right), & x>0\end{cases} \\
H(x)=\left\{\begin{array}{lll}
\exp \left(-|x|^{\alpha}\right), & x<0, \\
1, & x>0
\end{array}\right.
\end{gathered}
$$

donde la primera se identifica como la función de distribución Gumbel, la segunda como la Fréchet y la tercera como la función Weibull, y que en conjunto pueden representarse como la distribución generalizada del valor extremo (GEV) identificada como

$$
H(x)=\exp \left\{-\left(1+\xi \frac{x-\mu}{\beta}\right)_{+}^{-1 / \xi}\right\}
$$

y donde $\mu$ es el parámetro de ubicación, $\beta>0$ es el parámetro de escala y $\xi$ es el parámetro que da la forma a la función de distribủción.

Los tres tipos de distribuciones de probabilidad son recuperadas a partir de diferentes valores de $\xi:$ si $\xi \rightarrow 0$ se tiene una función de probabilidad Gumbel, 
si $\xi>0$ la función es Fréchet $\operatorname{con} \alpha=1 / \xi$ y si $\xi<0$ se trata de una Weibull $\operatorname{con} \alpha=-1 / \xi$.

\subsection{Modelo de picos sobre el umbral (POT)}

Sean $X_{1}, X_{2}, \cdots$ variables aleatorias idénticamente distribuidas con una función de probabilidad desconocida $F(x)=\operatorname{Pr}\left(X_{i} \leq x\right)$. La expresión

$$
1-F_{u}(y)=\operatorname{Pr}(X-u \geq y / X>u),
$$

donde $0 \leq y \leq x_{0}-u$, donde $x_{0} \leq \infty$ es el límite derecho de $F$ se define como la distribución de las pérdidas esperadas sobre un umbral $u$, la cual representa la distribución de probabilidad de las vaeiables aleatorias $X_{i}$ que exceden el umbral $u$. Utilizando el teorema de Bayes, la probabilidad acumulativa puede escribirse como

$$
1-F_{u}(y)=\frac{\operatorname{Pr}(X \geq u+y, X>u)}{\operatorname{Pr}(X>u)}=\frac{1-F(y+u)}{1-F(u)},
$$

donde la ecuación es expresada solo en función de distribución de la variable aleatoria cuyo comportamiento es desconocido.

Redefiniendo algunos de los términos de la expresión (7) se tiene que

$$
\begin{aligned}
1-F_{u}(y) & =\overline{F_{u}(y)}, \\
1-F(u) & =\overline{F(u)}
\end{aligned}
$$

y por tanto, la ecuación (7) puede escribirse entonces como

$$
\overline{F_{u}(y)} \overline{F(u)}=\overline{F(y+u)} .
$$

El punto siguiente es la evaluación de la expresión (9): en primer lugar, se procede con el término $\overline{F(u)}$, el cual se estima de forma empírica como el número de casos exitosos entre el total,

$$
\hat{F}(u)=\frac{1}{n} \sum_{i=1}^{n} I\left(X_{i}>u\right)=\frac{N_{u}}{n} .
$$

El cálculo de $\overline{F_{u}(y)}$ implica necesariamente, suponer una función de distribución de los excedentes, para lo cual se propone una distribución Pareto Generalizada (GPD), que se denota como

$$
F_{u}(y)=G_{\xi, \beta}(y)= \begin{cases}1-\left(1+\xi \frac{y}{\beta}\right)^{-1 / \xi}, & \xi \neq 0, \\ 1-\exp \left(-\frac{y}{\beta}\right), & \xi=0,\end{cases}
$$

donde $\beta>0$ y $y \geq 0$ cuando $\xi \geq 0$ y $0 \leq \mathrm{y} \leq-\beta / \xi$ cuando $\xi<0$. 
La distribución Pareto Generalizada puede ser identificada con diferentes funciones de probabilidad dependiendo del valor de sus parámetros: si $\xi>0$, la función de distribución es una Pareto; si $\xi=0$ se trata de una función de probabilidad exponencial y si $\xi<0$ se trata de una Pareto tipo II.

Finalmente, sustituyendo las ecuaciones (10) y (11) en la (9) se obtiene la expresión que permite evaluar las probabilidades de los excesos:

$$
\overline{\hat{F}(x)}=\overline{\hat{F}(y+u)}=\frac{N_{u}}{n}\left(1+\hat{\xi} \frac{y}{\hat{\beta}}\right)^{-1 / \hat{\xi}},
$$

donde $\hat{\xi}$ y $\hat{\beta}$ son estimaciones de los parámetros originales $\xi$ y $\beta$ y a partir de la cual se estima la función acumulativa de probabilidad, resultando

$$
\hat{F}(x)=\hat{F}(y+u)=1-\frac{N_{u}}{n}\left(1+\hat{\xi} \frac{y}{\hat{\beta}}\right)^{-1 / \hat{\xi}}
$$

Este último resultado puede ser utilizado para la medición de cuantiles de la distribución, herramienta útil en la administración de riesgos, particularmente en el cálculo del Valor en Riesgo, lo cual se desarrolla en la siguiente sección.

De acuerdo con Pickands (1975), la teoría clásica de eventos extremos es congruente con la desarrollada por el método POT en el sentido que, dada una función de probabilidad $F$, es factible aproximarla mediante una distribución Pareto generalizada si y solo si existen las constantes de normalización $a_{n}$ y $b_{n}$ y una distribución límite $H$, tal que la función límite clásica de eventos extremos se mantiene. En ese caso, si $H$ es una función generalizada de valor extremo, el parámetro $x$ es el mismo tanto en la generalizada de valor extremo como en la Pareto generalizada.

En este trabajo se utilizará el método de Picos sobre el umbral pues se desea utilizar todas las observaciones que excedan un umbral predeterminado, lo cual no es posible en los modelo de bloque máximo, modelo que además permitirá estimar la pérdida estimada condicional, medida de riesgo que se presenta a continuación.

\section{Administración de riesgos sistemáticos e intermediación financiera}

En los últimos años, en parte a la desregulación de los mercados financieros y a la integración mundial, muchas de las variables económicas se han caracterizado por mostrar una gran volatilidad, lo que ha motivado el desarrollo de herramientas para la administración de riesgos, las cuales han encontrado una aplicación natural en las autoridades financieras pues son responsables del monitoreo de los intermediarios financieros, instituciones que administran recursos de terceros y que por tanto deben de tener un marco regulatorio apropiado para disminuir el impacto de los riesgos sistémicos. ${ }^{1}$

1 Los riesgos sistémicos se refieren a las fallas de algunos participantes en el mercado que pueden "contagiarse" sobre otros participantes, magnificando la falla en un sistema financiero. 
Las fallas de estas regulaciones pueden generar desastres financieros de gran magnitud, como los protagonizados por Barings, Metallgesellschaft, Orange County y Daiwa entre otros, que son ahora contraejemplos del uso de las herramientas de coberturas de riesgos. ${ }^{2}$

En este sentido, los esfuerzos por crear marcos regulatorios de protección a los riesgos del mercado parten del acuerdo de Basilea de 1988, que representó el primer esfuerzo para crear un sistema de protección para los recursos de los ahorradores bancarios. La finalidad de estos marcos es el disminuir riesgos que puedan derivar en corridas bancarias o en un colapso del sistema de pagos.

En los acuerdos de Basilea posteriores se incluyeron otros elementos, tales como los requerimientos mínimos de capital, reglamentos para la diversificación de carteras, y una medición oficial del riesgo que representa una cartera de inversión: el Valor en Riesgo, el cual advierte sobre los recursos necesarios para cubrir las pérdidas ocasionadas por los portafolios de inversión de los bancos sobre un horizonte de tiempo y con un nivel de confianza definido.

Sin embargo, no son sólo los bancos quienes participan en este tipo de riesgos, lo son también, las casas de bolsa, las aseguradoras y las afianzadoras entre otros, principalmente por la naturaleza de sus negocios y al mismo tiempo, por la veloz y generalizada convergencia que está observándose entre estas instituciones a lo largo de los distintos mercados financieros.

En este escenario, la protección contra el riesgo sistémico para las aseguradoras y afianzadoras demanda un nuevo paradigma de regulación, que contemple además de los requerimientos de capital, herramientas para la prevención de crisis, la minimización del riesgo moral, la transparencia en sus operaciones y probablemente algunas reformas legales.

Técnicamente, la regulación respecto a los riesgos de mercado para evitar los riesgos sistémicos ha sido establecido por medio del cálculo del Valor en Riesgo $(V a R)$, que significa la posibilidad de cumplir con los peores escenarios respecto al riesgo de mercado con un grado de certidumbre de, generalmente, el $95 \%$ de los casos.

En las siguientes secciones se presenta la metodología general de medición del $V a R$ y la propuesta de cálculo a partir del método de picos sobre el umbral de la teoría de eventos extremos. Se presentará la medida del riesgo esperado excedente al $V a R$ y se ofrecerá un mecanismo dinámico para su estimación.

\subsection{Cálculo del Valor en Riesgo ( $V a R)$}

El Valor en Riesgo resume en un solo dato la pérdida máxima esperada sobre un horizonte de tiempo con cierto nivel estadístico de confianza. En términos generales, el $V a R$ es calculado como la diferencia entre el rendimiento esperado del portafolio de inversiones $(E(W))$ y el peor escenario posible $\left(W^{*}\right)$ que sucede con cierto nivel de confianza, es decir,

$$
\operatorname{VaR}(\text { Media })=E(W)-W^{*} .
$$

El $\mathrm{VaR}$ es entonces el monto esperado de pérdida en una inversión si el peor escenario resultara cierto, lo cual es relevante para tomar medidas de protección

2 Véase Jorion (1997) para estudios de caso de estas empresas. 
para ese resultado "catastrófico". En el contexto de la regulación el VaR es un parámetro del grado de riesgo que una empresa está asumiendo es sus inversiones y permite la cuantificación de las reservas que tendrán que constituirse para enfrentarla.

Cuando los rendimientos se distribuyen de acuerdo a una función de probabilidad normal, el $V a R$ adquiere una expresión sencilla y ampliamente conocida en los cursos de estadística,

$$
\operatorname{VaR}(M e d i a)=W_{0} \alpha \sigma \sqrt{\Delta t}
$$

donde $\alpha$ es el nivel de confianza, $W_{0}$ al valor del portafolio en el momento inicial, $\sigma$ es la desviación estándar de la muestra de datos y $\Delta t$ es el intervalo de tiempo.

\subsection{Cálculo del $V a R$ en el modelo de picos sobre el umbral}

A partir de la función de probabilidad estimada en la ecuación (13) para la modelación de los eventos extremos, el $V a R$ se calcula por medio de la inversa de la función de distribución, resultando

$$
V a R_{q}=u+\frac{\hat{\beta}}{\hat{\xi}}\left[\left(\frac{n}{N_{u}}(1-q)\right)^{-\xi}-1\right]
$$

donde $q$ representa el nivel de confianza asumido en la estimación del umbral, que debe ser mayor que $u$.

La aportación de este modelo de cálculo del $V a R$ es que mide con mayor precisión el peso de las colas de una distribución. Esto es particularmente útil en el caso de los rendimientos financieros, pues se identifican por tener distribuciones de colas pesadas (Jarrow y Roselfeld (1984), Venegas-Martínez (2001) entre otros), con lo cual el cálculo del $V a R$ con una distribución normal subestimará las colas de la distribución, lo que implicará un $V a R$ de mayor tamaño.

\subsection{Cálculo de la pérdida esperada condicional (PEC) en el modelo de eventos extremos}

Una medida que resulta útil en la práctica, es la pérdida esperada condicionada (PEC) que define la pérdida esperada una vez que el umbral determinado por el $V a R$ es superado. ${ }^{3}$ A diferencia del $V a R$, la pérdida esperada condicional si cumple con los axiomas de coherencia que definen una medida de riesgo financiero: subaditividad, monotonía, homogeneidad positiva y translación invariante (Artzner, et al (1997 y 1999), Acerbi y Tasche (2001) y Embrechts (2000) entre otros). ${ }^{4}$

3 A esta expresión también se le denomina Pérdida Esperada (Expected Shortfall o ES).

4 Para la profundización sobre las definiciones de coherencia recúrrase a Artzner, Delbaen, Eber y Heath (1997 y 1999). 
La pérdida esperada condicionada (PEC) es entonces una medida coherente que puede ser utilizada para medir la severidad de los riesgos y que se define como

$$
P E C_{q}=\mathrm{E}\left(X / X>V a R_{q}\right) .
$$

La PEC puede ser expresado en función del $V a R$ como

$$
\begin{aligned}
P E C_{q} & =V a R_{q}+\mathrm{E}\left(X-V a R_{q} / X>V a R_{q}\right) \\
& =\frac{V a R_{q}}{1-\xi}+\frac{\beta+\xi u}{1-\xi}
\end{aligned}
$$

donde la primera igualdad corresponde a la definición de la PEC a partir del VaR y la segunda corresponde a su definición a partir de la EVT.

\section{Aplicaciones en el caso del mercado mexicano de seguros}

Para demostrar las medidas del riesgo y la utilidad de la teoría del valor extremo en la industria de los seguros, se analizan dos casos correspondientes al mercado de seguros en México. En el primero se destaca la utilidad de las medidas de riesgo extremo en la administración de riesgos financieros en una aseguradora. En el segundo, se ofrece un análisis de los riesgos operativos de las carteras de seguros y la utilidad de la metodología planteada.

Entre las empresas aseguradoras, la teoría de los eventos extremos es ampliamente conocida en operaciones tales como incendio, inundación, terremoto, etc., eventos de una probabilidad baja pero de consecuencias económicas muy importantes para las empresas, eventos que pueden sin embargo suceder de manera recurrente ( 1 cada 100 días o una vez al año) cuando los casos de estudio corresponden a los riesgos financieros de la empresa o los riesgos operativos.

\subsection{Teoría de eventos extremos y los riesgos financieros de una ase- guradora}

\subsubsection{Antecedentes}

Una empresa de seguros es una institución que administra recursos de terceros comprometiéndose a devolver sumas aseguradas ante la ocurrencia de ciertos eventos contingentes, particularmente relacionados con la muerte, los accidentes, las enfermedades y las pérdidas de bienes físicos y hasta algunos intangibles.

Como cualquier intermediario financieros, los activos de las aseguradoras están constituidos en su gran mayoría por inversiones de los recursos de terceros, que representan obligaciones probables de pago en una fecha futura. Por lo que una situación de crisis de estas empresas representa la insolvencia, que imposibilite su operación.

Por lo tanto, para disminuir el riesgo de insolvencia, las empresas aseguradoras requieren de metodologías cada vez más precisas para la estimación de los riesgos financieros de sus inversiones, pues de esta rentabilidad depende su utilidad y la solvencia necesaria para hacer frente a sus obligaciones futuras.

Para abordar este caso se propone una medición del riesgo de mercado para el caso de la inversión de las reservas técnicas propuesta para el mercado mexicano, establecidos como las Reglas para la inversión de las reservas técnicas de 
las instituciones y sociedades mutualistas de seguros, cuya última modificación se publicó el día 6 de febrero del 2003 en el Diario Oficial de la Federación.

\subsubsection{Reglas para la inversión}

En estas reglas se determina que las reservas técnicas se invertirán con un límite de hasta $100 \%$ en instrumentos emitidos o respaldados por el Gobierno Federal; en valores emitidos y respaldados por instituciones de crédito, hasta el $18 \%$ y de hasta $7 \%$ por otros emisores. Está permitida además la inversión en activos de grupos, instituciones o sociedades que por su sector de actividad constituyan riesgos comunes, así clasificadas por la Bolsa Mexicana de Valores, por hasta un $10 \%$, pero si se trata del sector de la construcción el límite es $20 \%$. Está también permitida la inversión en activos o instrumentos que constituyan riesgos comunes, avalados por instituciones financieras y que mantengan nexos patrimoniales con la compañía de seguros, por una proporción de hasta el 5\%. Finalmente, la ley permite invertir hasta un $18 \%$ en activos o valores avalados o aceptados por sociedades relacionadas entre si.

Atendiendo a las restricciones por tipo de valores, títulos, bienes, créditos, reportos u otros activos, se permite hasta un $100 \%$ de inversión en instrumentos emitidos por el Gobierno Federal, 60\% en instrumentos privados y $30 \%$ por valores de otras instituciones diferentes a las anteriores.

También se imponen restricciones en cuanto al plazo de los instrumentos, destacándose que para las reservas de riesgo en curso, el límite mínimo a invertir en instrumentos de corto plazo es del $50 \%$; para las obligaciones pendientes de cubrir el límite es del 100\%; el de siniestros ocurridos y no reportados el límite es de $75 \%$ y el resto de reservas tienen límites del $20 \%$ o $30 \%{ }^{5}$

\subsubsection{Resultados financieros del mercado asegurador mexicano}

De acuerdo con el anuario estadístico 2001 de la Comisión Nacional de Seguros y Fianzas, para el año 2000, las inversiones constituyeron el $70 \%$ de los activos totales del sector, de las cuales las inversiones en instrumentos financieros representó el 91.06\%. Para el año 2001, las inversiones aumentaron a $73.08 \%$ del total de los activos y las inversiones en activos financieros representaron el 93.08\%. En el año 2002 el $73.7 \%$ de los activos totales correspondían a inversiones y en el 2003 esta cifra aumentó a $76.4 \%$, mientras que el $93.9 \%$ en 2002 y el $94.6 \%$ en 2003 de las inversiones totales estaban destinadas a inversiones en valores. Estas cifras muestran en un periodo de 4 años la importancia creciente de las inversiones en valores en los activos totales de las empresas de seguros.

Como lo muestra el Cuadro 1, las inversiones gubernamentales han venido en aumento a lo largo de estos años de análisis, pasando del 2000 al 2003 del $66.7 \%$ de las inversiones en valores al $76.5 \%$, años donde los derivados han tenido apenas un ligero incremento porcentual en su participación.

5 Las reglas fueron publicadas en el Diario Oficial de la Federación el día 18 de agosto del 2000 y modificadas el 13 de agosto del 2001 y el 6 de febrero del 2003. 
Cuadro 1. Instrumentos de inversión del mercado asegurador en proporción, 2000-2003.

Instrumentos de

\begin{tabular}{cccccc} 
inversión & 2000 & 2001 & 2002 & 2003 & Promedio \\
\hline Gubernamentales & $66.71 \%$ & $72.88 \%$ & $74.75 \%$ & $76.47 \%$ & $72.70 \%$ \\
Privados & $33.29 \%$ & $27.12 \%$ & $25.25 \%$ & $23.53 \%$ & $27.30 \%$ \\
Derivados & $0.00 \%$ & $0.01 \%$ & $0.01 \%$ & $0.03 \%$ & $0.01 \%$ \\
\hline
\end{tabular}

Fuente: Anuarios Estadísticos de Seguros y Fianzas de los años respectivos.

En el estado de resultados, las utilidades financieras representaron en el 2000 una proporción del $3.79 \%$ de las primas emitidas, para el 2001 representaron el $8.17 \%$, para el 2002 el $7.47 \%$ y el $10.45 \%$ en el 2003 . En ambos años los resultados financieros mejoraron de forma muy importante las pérdidas operativas registradas, llevando a las empresas a niveles importantes de utilidades antes de impuestos. ${ }^{6}$

Por otro lado, en el lado de los pasivos, se observa que las reservas técnicas representaron en el 2000 el $72.4 \%$ de los activos totales, en el 2001 el $75 \%$, aumentando ligeramente a $75.4 \%$ en el 2002 y disminuyendo en el 2003 a $72.2 \%$. En función de los pasivos, las reservas representaron el $86 \%$ de los pasivos totales en los 2000 y 2001 y $87 \%$ en 2002 y 2003, las cuales deben ser invertidas siguiendo las reglas emitidas por la CNSF.

En promedio de los 4 años, el $71 \%$ de la totalidad de las reservas corresponden a reservas de riesgos en curso, el $12 \%$ corresponden a reservas por obligaciones pendientes de cubrir, el 9\% lo constituyen reservas de previsión y contingencia, el $5 \%$ son reservas para riesgos catastróficos y el $4 \%$ por siniestros ocurridos y no reportados.

\subsubsection{Supuestos para la medición del riesgo financiero}

Para hacer una medición del riesgo financiero se enuncian ahora algunos supuestos simplificadores que permitirán abordar el complejo problema de inversión de las reservas técnicas de las empresas aseguradoras.

Se asumirá entonces, como una aproximación a la manera de invertir del conjunto de empresas del sector asegurador, un portafolio compuesto en un $73 \%$ por instrumentos gubernamentales y en un $27 \%$ por instrumentos privados, de los cuales el $74 \%$ serán de renta fija y el restante de renta variable. En ningún momento las inversiones de activos deberán superar el 100\% para los gubernamentales, $18 \%$ los privados avalados por instituciones de crédito y $7 \%$ el resto. ${ }^{7}$

6 De forma concreta se puede decir que las inversiones salvan a las aseguradoras de las pérdidas operativas.

7 Todas estas cifras consideradas supuestos se han obtenido como promedios de los años considerados. 
Al observarse que las reservas técnicas corresponden casi exactamente a las inversiones que mantienen las empresas de seguros, se asume que las inversiones corresponden a la totalidad de reservas y que éstas inversiones deben regirse por las reglas emitidas por la CNSF.

Para cumplir con las restricciones de liquidez, al menos el $54 \%$ de las reservas debe estar invertido en instrumentos de corto plazo (menores a 1 año) y el restante puede ser invertido a plazos mayores. En cualquier caso, los rendimientos ofrecidos por los instrumentos en sus diferentes plazos serán quienes indiquen si se cumplen los mínimos o no.

Para una aproximación de los instrumentos gubernamentales se utilizará la variable que expresa la tasa diaria de fondeo gubernamental, que es un índice de diferentes instrumentos de gobierno a un plazo diario. Como estimador de los diferentes instrumentos privados de renta variable, se utilizará el IPC, que sirve en múltiples ocasiones como un indicador del mercado accionario en México. Como estimador de los instrumentos privados de renta fija se utilizará la tasa de fondeo bancaria, tasa menor a la que se obtiene en los mercados financieros por instrumentos de deuda privados. Las proporciones de cada uno de estos activos se muestran en la Cuadro 2.

Cuadro 2. Instrumentos utilizados en el portafolio simulado.

\begin{tabular}{ccc} 
Activo & Características & $\begin{array}{c}\text { Proporción en el } \\
\text { mercado mexicano } \\
\text { de seguros }\end{array}$ \\
\hline $\begin{array}{c}\text { Tasa de fondeo } \\
\text { gubernamental }\end{array}$ & $\begin{array}{c}\text { Gubernamental de } \\
\text { corto plazo }\end{array}$ & $73 \%$ \\
\hline IPC & $\begin{array}{c}\text { Privado de corto plazo } \\
\text { Renta Variable }\end{array}$ & $20 \%$ \\
\hline $\begin{array}{c}\text { Tasa de fondeo } \\
\text { bancaria }\end{array}$ & $\begin{array}{c}\text { Privado de corto plazo } \\
\text { Renta Fija }\end{array}$ \\
\hline
\end{tabular}

\subsubsection{Comportamiento de los instrumentos financieros}

En principio, se mostrarán gráficas de los 3 instrumentos utilizados para modelar el comportamiento de la cartera de inversiones de una aseguradora, los cuales se presentan con una frecuencia diaria en las Gráficas 1a, 1b y 1c en el periodo del 3 de enero del 2000 al 18 de febrero del 2005, periodo en el que los instrumentos han pasado por periodos de gran volatilidad.

Para efectos de la medición de los rendimientos y de la estimación de las características, además de la medición más precisa de las colas de la distribución de probabilidad de las variables se utilizará un conjunto más amplio de datos, desde el 4 de enero de 1999 hasta el 18 de febrero de 2005, de esta manera el $5 \%$ de los casos estaría representado por una inuestra de 78 datos aproximadamente. En el Cuadro 3 se presentan las características de estas variables. La columna que se titula portafolio corresponde al portafolio de inversiones propuesto con los pesos expresados en el Cuadro 2. 
Gráfica 1a. Rendimiento diario del instrumento de renta fija gubernamental del 5-01-99 al 18-02-05.

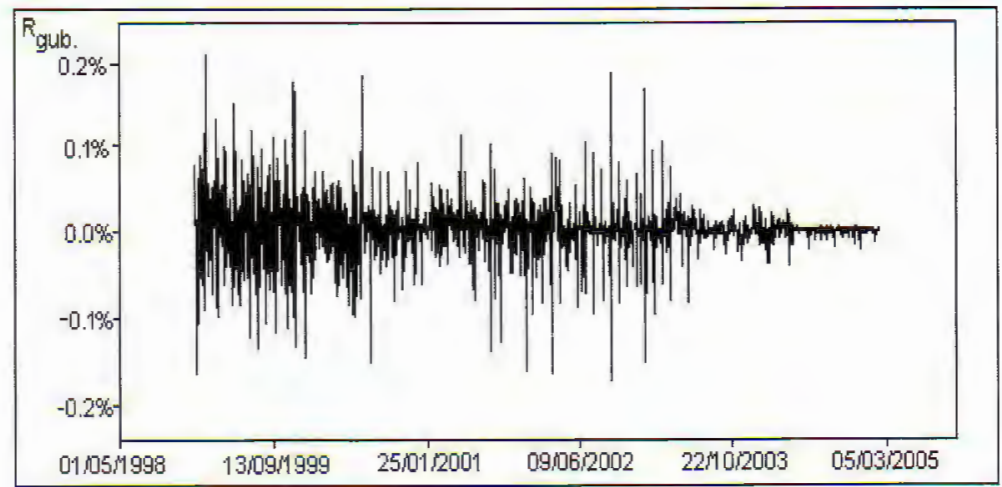

Gráfica 1b. Rendimiento diario del instrumento privado de renta fija del 5-01-99 al 18-02-05.

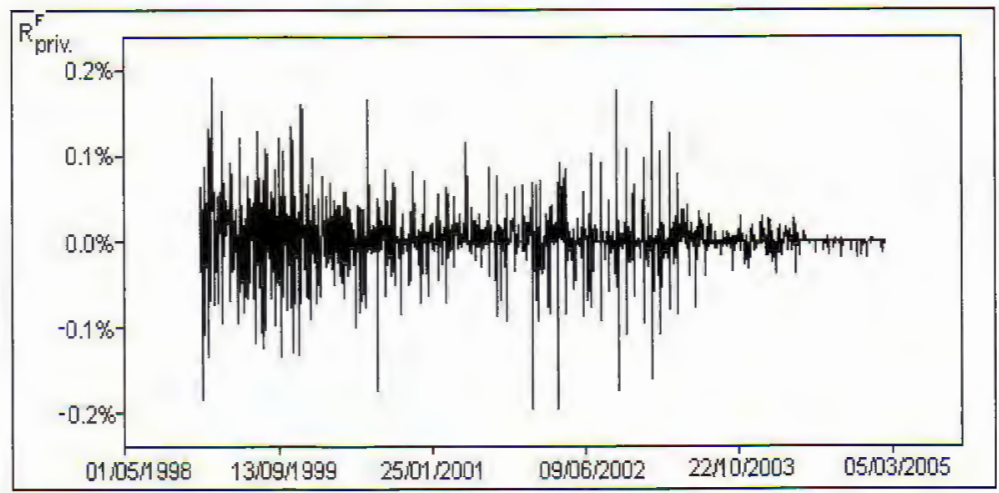

Gráfica 1c. Rendimiento del instrumento privado de renta variable del 5-01-99 al 18-02-05.

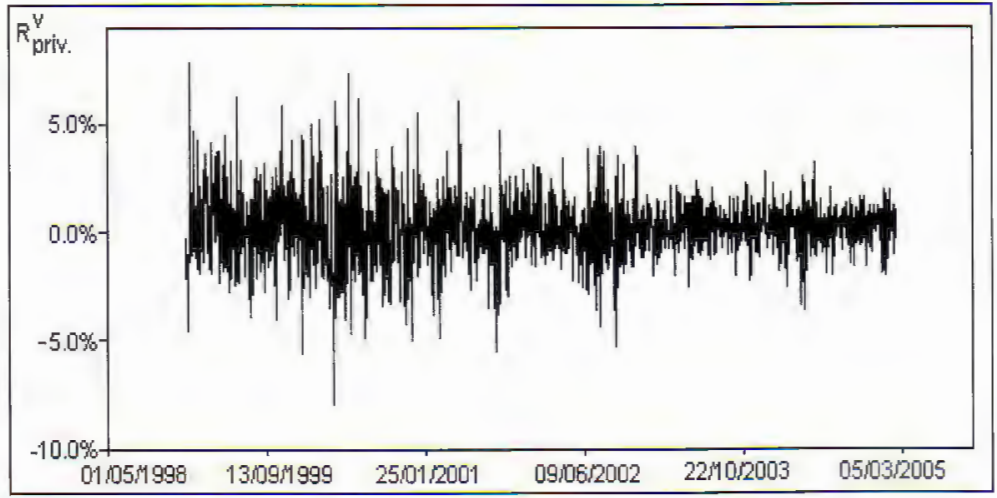


Cuadro 3. Resumen de datos estadísticos.

\begin{tabular}{ccccc}
\hline Estadístico & $\begin{array}{c}\text { Papel } \\
\text { gub. }\end{array}$ & $\begin{array}{c}\text { Privado } \\
\text { renta fija }\end{array}$ & $\begin{array}{c}\text { Privado renta } \\
\text { variable }\end{array}$ & Portafolio \\
\hline Media & $0.001062 \%$ & $0.001145 \%$ & $0.096073 \%$ & $0.007537 \%$ \\
Mediana & $0.000773 \%$ & $0.000000 \%$ & $0.092788 \%$ & $0.008269 \%$ \\
Desv. estándar & $0.033963 \%$ & $0.034352 \%$ & $1.521623 \%$ & $0.117219 \%$ \\
Asimetría & -0.170544 & -0.272676 & 0.193492 & 0.163308 \\
Kurtosis & 9.592442 & 10.471910 & 5.552416 & 6.180161 \\
Jarque-Bera & 2808.86 & 3617.80 & 428.88 & 658.76 \\
Probabilidad & 0.000000 & 0.000000 & 0.000000 & 0.000000 \\
\hline
\end{tabular}

Es de particular interés la prueba de normalidad de Jarque-Bera, que muestra que los datos difícilmente pueden describirse como una función de probabilidad normal. Si a ese resultado se le incorpora la condición de exceso de kurtosis, el resultado es un proceso de colas pesadas, descripción que puede ser mejorada con la distribución de riesgos extremos.

La Gráfica 2 muestra las gráficas Q-Q para la distribución normal para los 3 instrumentos financieros considerados, donde se observa que en las colas de la distribución es principalmente donde existen mayores dificultades para poder ajustarse a ésta, indicando entonces un mayor peso con respecto al asignado por la función de probabilidad normal.

Gráfica 2. Gráficas Q-Q para la función de probabilidad normal.
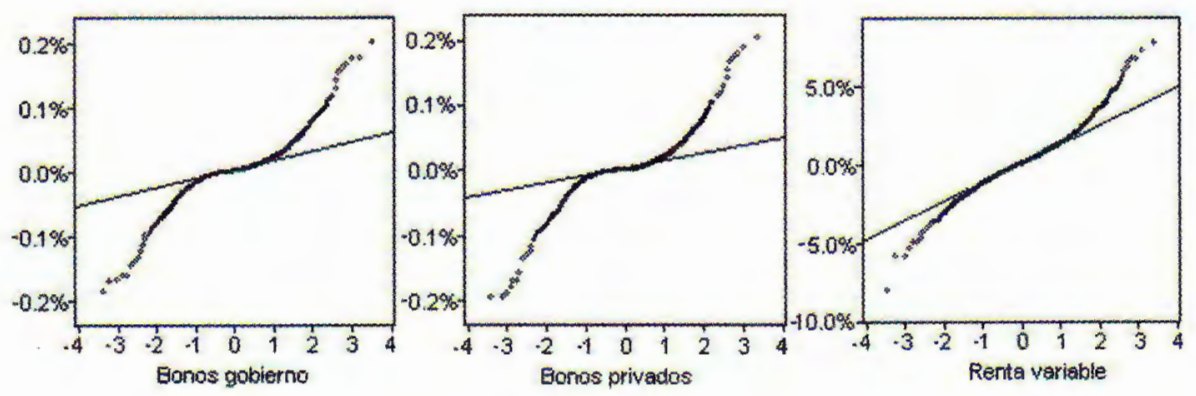

\subsubsection{Estimación del peor escenario y pérdida esperada condicional}

Para un total de 1,547 observaciones, el peor escenario con un nivel de confianza del $95 \%$ estará dado por un conjunto de 77 observaciones sobre un umbral $u$, el cual, bajo la condición de un mayor peso en las colas de las distribuciones de los activos que conforman el portafolio y asumiendo que se distribuyen como una $G P D$, se pueden estimar mediante las expresiones (15) y (17).

Se estimarán dos estadísticos, un VaR asumiendo una distribución normal y un $V a R$ asumiendo que las colas tienen una distribución Pareto Generalizada lo 
cual, como se ha mostrado, es más congruente con los datos empíricos mostrados en el portafolio de inversión. El periodo de análisis es del 5 de enero del 1999 al 18 de febrero de 2005.

Se comienza entonces caracterizando el comportamiento del portafolio de inversión definido por los activos mencionados en las ponderaciones propuestas en el Cuadro 2, cuyo comportamiento histórico se muestra en la Gráfica 3 y sus medidas estadísticas típicas se presentan en el Cuadro 2.

Las ponderaciones del portafolio no son ajustadas a lo largo del tiempo, siempre permanecen fijas de acuerdo a las ponderaciones establecidas inicialmente. Se utiliza entonces un modelo ARIMA-GARCH para describir el comportamiento histórico de sus rendimientos del portafolio a partir de su propia historia y con presencia de una heteroscedasticidad condicional.

El uso de modelos GARCH (Engle (1982) y Bollerslev (1986)) es propio de las series financieras, caracterizadas por una abundante cantidad de datos medidos en una frecuencia muy alta. Por su parte, los modelos EGARCH (Nelson (1991)) se identifican con un proceso de heteroscedasticidad condicional exponencial, pues el logaritmo de la varianza condicional es la que se condiciona con logaritmos de las varianzas pasadas y con cocientes del error-desviación estándar en forma absoluta y en forma natural, expresando con ello los efectos asimétricos que tienen los rezagos. Finalmente, los modelos ARCH-M (Engle, Lilien y Russell (1987)) incoporan explícitamente en la ecuación de tendencia de la variable explicada el efecto de la varianza, modelo utilizado en finanzas donde los resultados esperados de los rendimientos están afectados por el grado de riesgo.

Una vez efectuada la estimación del modelo ARIMA-GARCH, expresado como

$$
\begin{aligned}
\Delta R_{p, t}= & -1.26 E^{-5}+0.082463 \Delta R_{p, t-1}-0.066558 \Delta R_{p, t-2} \\
& \left(4.43 E^{-6}\right) \quad(0.029349) \\
- & 0.057314 \Delta R_{p, t-5}-0.961623 \varepsilon_{t-1}+5.452862 \sigma_{t}^{2}+\varepsilon_{t}, \\
& (0.020364) \quad(0.007596) \\
\ln \left(\sigma_{t}^{2}\right)= & -5.281281+0.635238 \ln \left(\sigma_{t-1}^{2}\right)+0.409834\left|\frac{\epsilon_{t-1}}{\sigma_{t-1}}\right| \\
& (0.075666)(0.003228) \\
- & 0.126118 \frac{\epsilon_{t-1}}{\sigma_{t-1}} .
\end{aligned}
$$

en donde se aprecia que se trata de un modelo diferenciado (raíz unitaria) y se ajustó por medio de un modelo ARCH-M donde el modelo de la varianza condicional es exponencial (EGARCH), se calculan los residuos, los cuales tampoco se ajustan a una función de probabilidad normal, verificado por medio del estadístico de Jarque-Bera con valor de 321.56 que se asocia con una probabilidad de 0 se asemejarse a una normal. Los términos en paréntesis de la ecuación (18) corresponden a las desviaciones estándar de los estadísticos. 
Como conclusión de la estimación del ARIMA-EGARCH se puede decir que el proceso de rendimientos del portafolio se identifica con un proceso estocástico con volatilidad autorregresiva estocástica, donde $\varepsilon_{t}$ es el término estocástico del proceso de rendimientos, caracterizado como un proceso con media cero, varianza unitaria y función de densidad de probabilidad $f_{Z}(z)$, que no sigue una probabilidad normal pues presenta mayor peso en las colas y una curtosis mayor que la correspondiente a una función de distribución normal (5.24).

Se tiene entonces un modelo que predice el comportamiento de los rendimientos del portafolio hipotético con las composición propuesta anteriormente, el cual logra predecir con cuestionable precisión su comportamiento (45\%). El resto se explica por efectos aleatorios, no predecibles, de los rendimientos, los cuales se desea al menos caracterizar con una función de probabilidad para prevenirse de escenarios altamente riesgosos por representar grandes pérdidas. Entonces, de ahora en adelante, el análisis se centrará en los errores del modelo ARIMA-GARCH en un intento por tipificar su comportamiento.

Gráfica 3. Redimiento del portafolio de inversión del 05-01-99 al 18-06-05.

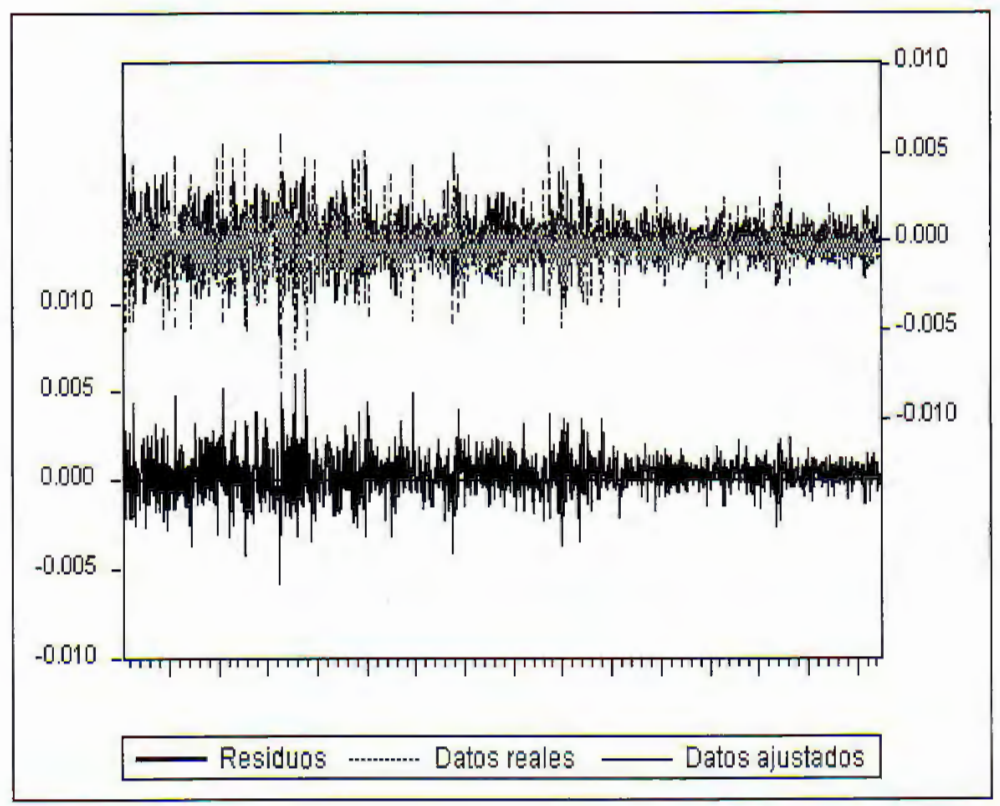

En el estudio de la distribución de las desviaciones grandes hacia la izquierda, las peores pérdidas, se desarrolla un ajuste por medio de una función de probabilidad Pareto Generalizada, por ser una distribución que ofrece una solución al caso de las colas pesadas. En la Gráfica 4 se observa el ajuste que se consigue cuando se consideran las 77 peores pérdidas diarias, es decir, el $5 \%$ de los casos.

Esta gráfica indica que el umbral, $u$, se determina en un valor de -0.00195 y, de acuerdo a la expresión (15), el Valor en Riesgo es $V a R_{0.99}^{E V T}=-0.58747 \%$ 
del rendimiento diario. Los parámetros ajustados para la GPD son $\hat{\xi}=0.127$ y $\hat{\beta}=0.000637$.

Por su parte, una distribución normal generaría un $V a R_{0.99}^{N}$ de $-0.2739 \%$, que comparado con el ofrecido por el EVT es mayor, mostrando así la presencia de colas pesadas en la distribución de los rendimientos negativos del portafolio de inversiones. Este resultado es muy relevante, pues las colas de la distribución normal, al ser más ligeras, presentan una mayor concentración alrededor de la media, con lo cual los intervalos generados son menores que los correspondientes a las distribuciones de colas pesadas, subestimando así las probabilidades pérdidas grandes.

La pérdida esperada condicional, de acuerdo a los resultados mostrados en el párrafo anterior y de acuerdo con la expresión (17) sería de $P E C_{0.99}=$ $-0.77428 \%$, la cual indica el monto esperado de pérdida sobre el umbral $u$ que corresponde a la peor pérdida posible, con una confianza del $99 \%$ sobre el rendimiento diario.

Gráfica 4. Distribución de probabilidad de las pérdidas sobre el umbral.

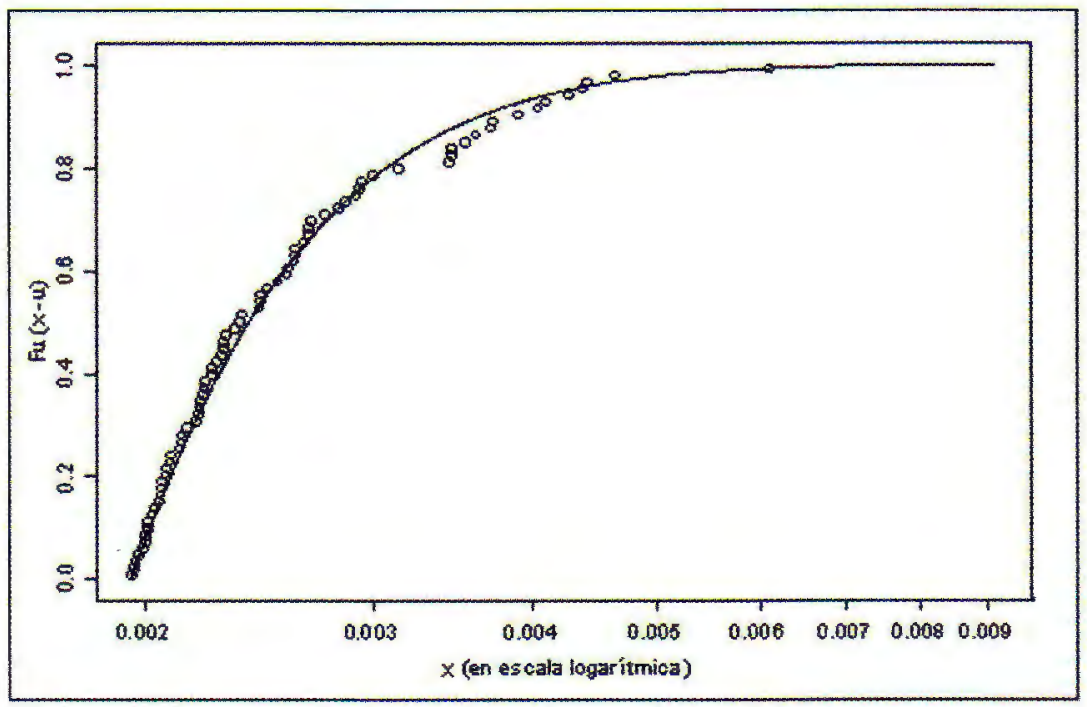

Finalmente, este modelo puede ser utilizado para estimar los valores del $V a R$ y de $P E C$ dinámicamente (McNeil (1999), McNeil y Frey (2000)), utilizando el modelo propuesto en la siguiente forma: si el comportamiento de los activos sigue un modelo estocástico del tipo mostrado en la ecuación (18), es posible demostrar que el $V a R$ puede ser estimado dinámicamente en cada periodo de acuerdo a las ecuaciones

$$
\begin{aligned}
V a R_{p, t} & =\mu_{t}+\sigma_{t} V a R_{p,(t-1),} \\
P E C_{t} & =\mu_{t}+\sigma_{t} P E C_{t-1},
\end{aligned}
$$

donde $\mu_{t}$ representa la tendencia del proceso que siguen los rendimientos del portafolio y $\sigma_{t}$ representa su desviación estándar. 
Como se ha probado, la tendencia del proceso de rendimientos sigue un proceso ARIMA donde la tendencia es afectada por la volatilidad (ARCH-M), y la volatilidad sigue un proceso GARCH exponencial (EGARCH), lo que define los parámetros $\mu_{t}$ y $\sigma_{t}$, resultando de la forma

$$
\begin{aligned}
\mu_{t} & =\alpha_{0}+R_{p, t-1}+\alpha_{1} \Delta R_{p, t-1}+\alpha_{2} \Delta R_{p, t-2}+\cdots+\beta \sigma_{t-1}^{2}+u_{t}+\gamma_{1} u_{t-1}+\ldots \\
\sigma_{t}^{2} & =\exp \left(\delta_{0}+\delta_{1} \log \left(\sigma_{t-1}^{2}\right)+\delta_{2}\left|\frac{u_{t-1}}{\sigma_{t-1}}\right|+\delta_{3} \frac{u_{t-1}}{\sigma_{t-1}}\right)
\end{aligned}
$$

cálculos que deberán efectuarse diariamente para poder pronosticar el nuevo $V a R$ y del $P E C$ en los periodos posteriores.

\subsection{Teoría de eventos extremos y riesgos operativos de una asegura- dora}

De manera teórica el proceso de reclamaciones de una compañía de seguros es modelado por medio de los procesos de ruina, el cual describe la manera en la que las reclamaciones van llegando en cada momento obedeciendo a una función de probabilidad definida. De la misma manera la llegada de las primas puede modelarse como un proceso estocástico.

La manera más sencilla de describir el comportamiento dinámico del monto de las reclamaciones es por medio de una función de distribución Poisson para el número de reclamaciones y una función de probabilidad $f(x)$ para el monto unitario de reclamación. Conjuntamente estas funciones forman un proceso Poisson compuesto.

La función $f(x)$ de reclamaciones unitarias, en el caso de los riesgos catastróficos, es identificada con una Pareto Generalizada (GPD); otros casos pueden considerar la distribución $X^{2}$, la $F$ o una distribución empírica.

El objetivo de esta sección es probar que en los seguros la teoría de riesgos extremos puede utilizarse en eventos que no necesariamente se clasifican como catastróficos. Tal es el caso de los gastos médicos mayores ( $S G M M$ ) que ofrecen cobertura por los gastos médicos imprevistos, que pueden generar desequilibrios financieros a una familia que no cuenta con esta protección.

Por el lado de la aseguradora, los gastos derivados de un exceso de reclamaciones en determinado periodo puede disminuir las utilidades de forma inportante, más si ésta es combinada con una baja en tasas de interés, como se ha estudiado en la sección anterior.

\subsubsection{Comportamiento histórico de las reclamaciones}

El conjunto de datos utilizados para mostrar un proceso normal de reclamaciones es de 6 años con una frecuencia mensual, lo cual significan 72 observaciones. Como el proceso de reclamaciones puede descomponerse en dos variables relativamente independientes, número y monto unitario de la reclamación, se muestran en la Gráfica 5 ambos procesos en una frecuencia mensual, describiendo el número de reclamaciones un comportamiento creciente a lo largo del periodo mientras que el monto unitario de las reclamaciones en pesos constantes presenta un comportamiento decreciente. 
En términos estadísticos, las reclamaciones no siguen una función de distribución normal, lo cual puede apreciarse claramente en la Gráfica 6 donde se ha presentado el histograma de frecuencias del monto reclamado para un subconjunto de las observaciones presentadas en la Gráfica 5 y para el cual se presenta una media de $\$ 15,035$, desviación estándar de $\$ 32,923$, coeficiente de asimetría de 10.12 y kurtosis de 172.62 , lo cual está bastante lejano de los parámetros de una densidad normal. En este subconjunto se cuenta con 21,270 datos y corresponde a un periodo de 6 meses.

Gráfica 5. Número y monto unitario de las reclamaciones mensuales.

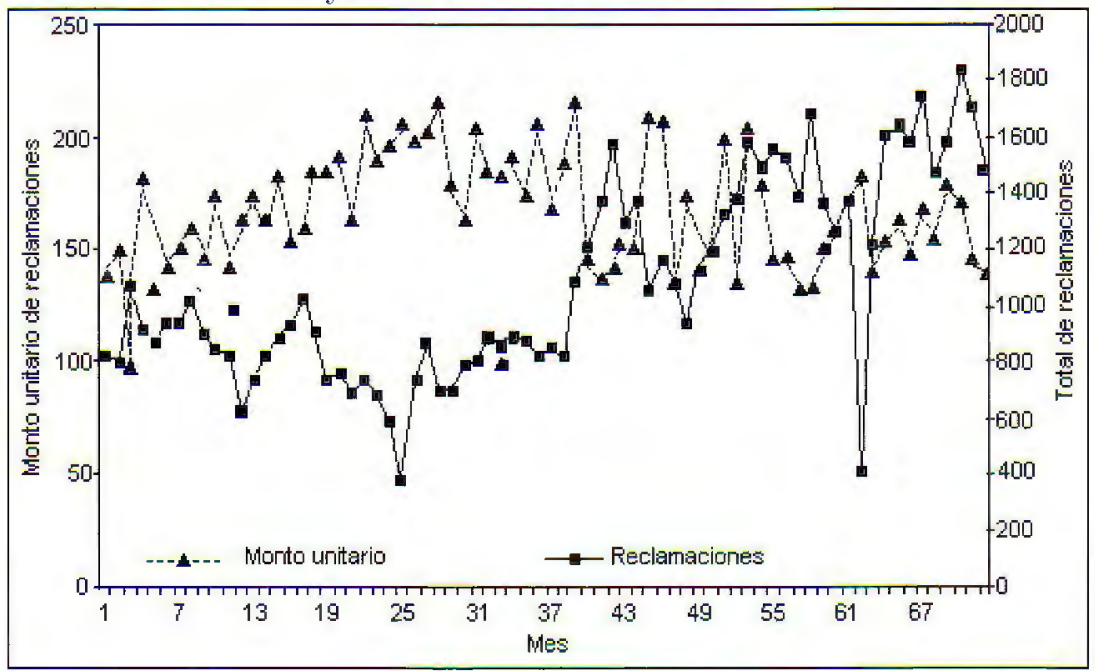

A partir del mismo conjunto de datos presentados en la Gráfica 6 se ofrece una descripción estadística por decil de la totalidad de las reclamaciones en dicho periodo, que como es de esperarse, en los primeros deciles la media y desviación estándar

Gráfica 6. Histograma de las reclamaciones en 6 meses.

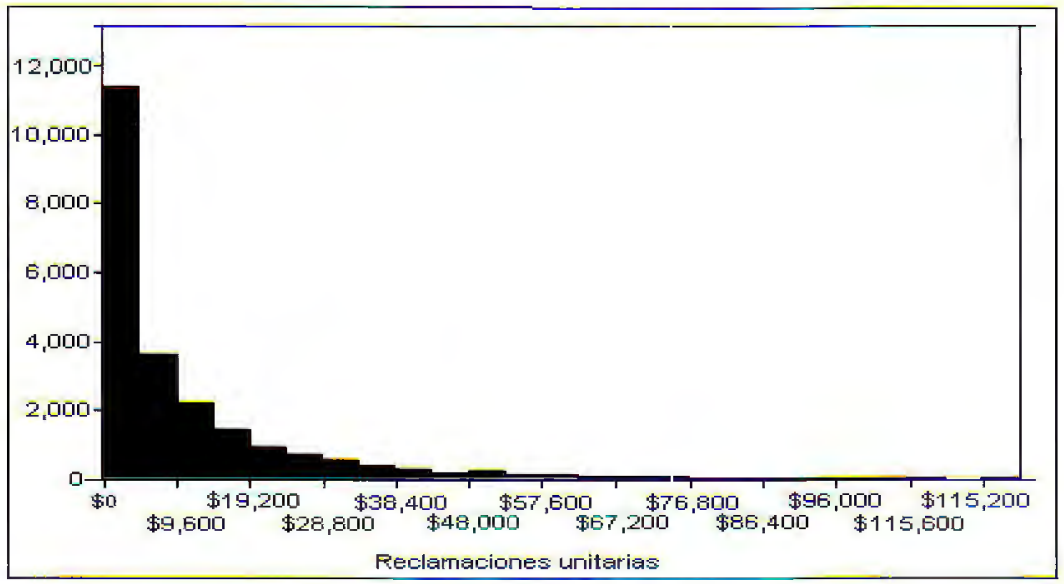


La situación que más preocupa a las aseguradoras es el riesgo de insolvencia, el cual se define como la imposibilidad por pagar las reclamaciones de sus asegurados ya sea por no poseer la liquidez suficiente, por algún siniestro colectivo que implica muchas reclamaciones y grandes sumas de dinero o por algún otro suceso que signifique que no se cuenta con los recursos necesarios para enfrentar las obligaciones con sus clientes.

Cuadro 4. Fstadística descriptiva por decil del monto de reclamaciones.

\begin{tabular}{cccccc}
\hline Percentil & $\begin{array}{c}\text { Limite del } \\
\text { percentil }\end{array}$ & Pronedio & $\begin{array}{c}\text { Desviación } \\
\text { Estándar }\end{array}$ & Asimetría & Kurtosis \\
\hline $10 \%$ & 830.04 & 504.48 & 195.40 & $(0.31)$ & $(0.71)$ \\
$20 \%$ & $1,556.85$ & $1,174.59$ & 210.01 & 0.20 & $(1.15)$ \\
$30 \%$ & $2,548.92$ & $2,017.61$ & 289.02 & 0.16 & $(1.19)$ \\
$40 \%$ & $3,900.98$ & $3,173.54$ & 383.27 & 0.17 & $(1.14)$ \\
$50 \%$ & $5,993.65$ & $4,864.45$ & 614.91 & 0.17 & $(1.19)$ \\
$60 \%$ & $8,955.99$ & $7,348.30$ & 846.96 & 0.20 & $(1.14)$ \\
$70 \%$ & $13,182.02$ & $10,882.43$ & $1,206.06$ & 0.17 & $(1.16)$ \\
$80 \%$ & $20,108.20$ & $16,279.41$ & $1,986.63$ & 0.21 & $(1.14)$ \\
$90 \%$ & $34,868.89$ & $26,521.57$ & $4,189.24$ & 0.28 & $(1.06)$ \\
$100 \%$ & $1,019,311.98$ & $77,515.09$ & $76,671.11$ & 5.05 & 35.90 \\
Total & & $15,032.16$ & $32,923.84$ & 10.12 & 172.62 \\
\hline
\end{tabular}

En el Cuadro 4 se presenta la estadística descriptiva de las observaciones del subconjunto de análisis, las cuales permiten observar el incremento tanto de los promedios como de las desviaciones estándar, mas no de su cociente, el cual es estable entre 5 y 10 excepto en el primero y último decil, en los cuales la razón es 2.58 y 1.01 respectivamente, mostrando el alto grado de dispersión relativa en los extremos de la distribución.

También en el Cuadro 4 puede apreciarse como la asimetría y la kurtosis son relativamente estables y uniformes a lo largo de la distribución, pero no para el último decil, el de las grandes reclamaciones, donde claramente se dan incrementos en estos parámetros. Lo que estos resultados permiten comprender es entonces el diferente comportamiento que presentan las colas de las distribución de reclamaciones para una compañía de seguros en sus contratos de gastos médicos mayores, con lo cual se justifica un tratamiento diferente para las colas y en este trabajo se desarrollará por medio de la teoría de eventos extremos.

\subsubsection{Análisis de la cola derecha de la distribución de las reclamacio- nes}

Ahora se desea estimar el comportamiento de la cola derecha de la distribución de reclamaciones del periodo de 6 meses que se ha descrito en la sección anterior ajustando a una distribución Pareto Generalizada mediante el método de picos sobre el umbral.

En este análisis se presenta en primer lugar el comportamiento de las colas de la distribución cuando se comparan con una función de probabilidad 
Pareto Generalizada, para el 5\% de las mayores reclamaciones conjunto de observaciones descritas y a las cuales corresponden los parámetros $u=55,000$, $\hat{\xi}=0.506$ y $\hat{\beta}=32,986.26$. Este comportamiento se describe en la Gráfica 7, que muestra en a) el comportamiento de los excedentes al umbral de acuerdo con una GPD y en b) la cola de la distribución en escala logarítmica.

Gráfica 7. Ajuste de las reclamaciones de mayor tamaño en SGMM.

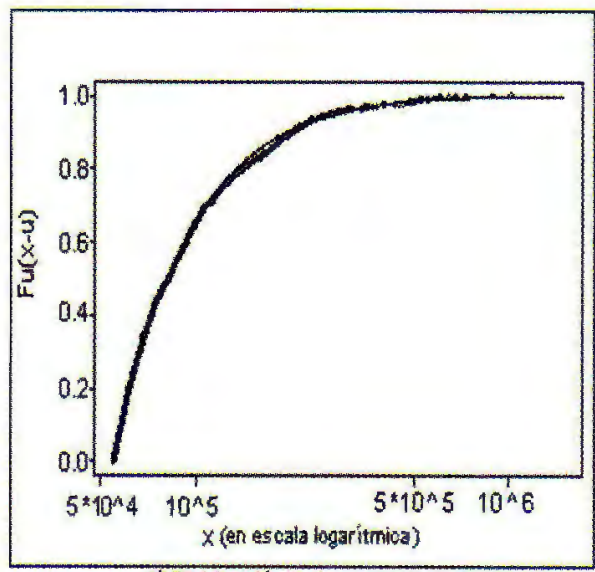

a) Distribucion de los excedentes

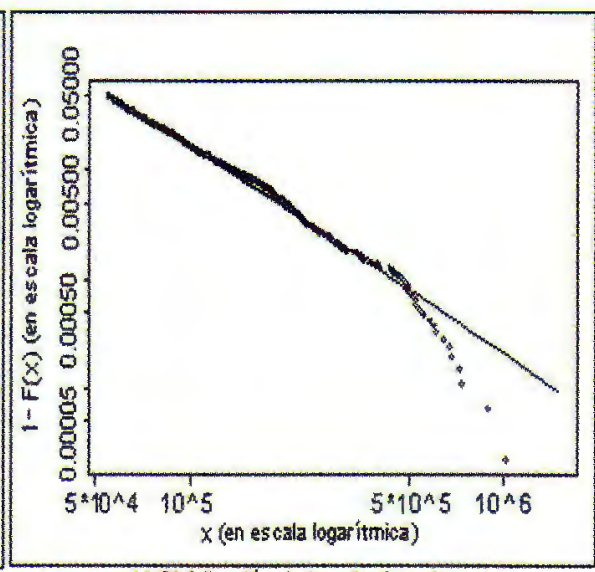

b) Oistribucion de la cola derecha

\subsubsection{Construcción del Valor en Riesgo y pérdida esperada condicio- nal de una cartera de seguros con la teoría del valor extremo}

Cuando se fija un umbral de 55,000, aún exceden aproximadamente el $5 \%$ de las observaciones ese monto, valor para el cual es posible construir a partir de la teoría del valor extremo y con el concepto de Valor en Riesgo, un mecanismo dinámico que permita proteger a la empresa de seguros de grandes reclamaciones que pongan en riesgo su solvencia.

A partir de las ecuaciones (15) y (17) es factible el cálculo del Valor en Riesgo y la pérdida esperada condicional a partir de los parámetros ajustados por la distribución Pareto Generalizada para la distribución de reclamaciones unitarias, la cual resulta ser, considerando un nivel de confianza $q$ de 0.975 , por ejemplo, define un valor límite de 83,547 y una pérdida esperada condicional de 292,233 por sobre el VaR.

Dado que el cálculo del $V a R$ y la $P E C$ definidos hasta este momento son válidos solamente para una reclamación, es necesario el paso hacia la medición del riesgo de la cartera total, que de acuerdo con el modelo de riesgo colectivo se compone además del riesgo del monto de las reclamaciones del riesgo de la cantidad de ellas en un periodo, es decir,

$$
S_{T}=X_{1}+X_{2}+\cdots+X_{N},
$$

donde $S_{T}$ es el monto total de reclamaciones, $X_{i}$ es la variable aleatoria que representa la reclamación total y $N$ el número de reclamaciones en el periodo 
de análisis. Por lo tanto, lo que ahora interesa es medir la probabilidad no condicional

$$
\mathrm{P}\left(S_{T}>V a R_{q}\right)=\mathrm{P}\left(X_{1}+X_{2}+\cdots+X_{N}>V a R_{q}\right)
$$

y construir, a partir de la teoría del valor extremo, el umbral adecuado sobre el que la distribución de los excedentes tendrá un comportamiento acorde a la Distribución Pareto Generalizada.

Sin embargo, ahora se tienen dos variables que influyen en el exceso del umbral para las reclamaciones totales: el número de reclamaciones y el monto unitario de cada una de éstas. El análisis de los excesos de las reclamaciones totales sobre el umbral $u$ debe considerar entonces ambos comportamientos. Debido a la poca cantidad de datos que en esta frecuencia se pueden obtener no es posible el ajuste de las colas de la distribución, por lo que se tendrá que deducir del comportamiento individual de las reclamaciones.

El problema de la suma de variables aleatorias en una cartera de seguros ha sido estudiado ampliamente y una posible solución se obtiene a partir del suponer que el número de reclamaciones obedece a una función de probabilidad Poisson, definiendo así la función de densidad Poisson compuesta, cuya expresión es

$$
\begin{aligned}
\mathrm{P}_{\mathrm{r}}\left(S_{T^{2}} \geq x\right) & =1-F(x) \\
& =\sum_{n=0}^{\infty} \mathrm{P}_{\mathrm{r}}\left(X_{1}+X_{2}+\cdots+X_{N} \geq x \mid N=n\right) \mathrm{P}_{\mathrm{r}}(N=n) \\
& =\sum_{n=0}^{\infty} \mathrm{P}_{\mathrm{r}}\left(X_{1}+X_{2}+\cdots+X_{n} \geq x \mid N=n\right) \frac{e^{-\lambda} \lambda^{n}}{n !}
\end{aligned}
$$

donde como puede observarse, la probabilidad condicional de que la suma de las reclamaciones no exceda el umbral $x$ cuando el número de reclamaciones es $n$ es una convolución, que solo puede ser resuelta cuando se conoce la densidad de la severidad de las reclamaciones $X_{i}, i=1,2, \ldots n$. De esta manera, el cálculo requerido en la expresión (22) puede ser estimado a partir de (23) para el valor de $x$ igual al $V a R_{q}$ una vez que $q$ sea definido.

Por otro lado, el $V a R$ de un portafolio puede estimarse como una suma de los $V a R$ individuales, lo cual se expresa como

$$
\begin{aligned}
\operatorname{VaR}\left[X_{1}+X_{2}+\cdots+X_{N} \mid N=n\right] & =\left[\sum_{i=1}^{n} V_{a} R^{2}\left(X_{i}\right)\right]^{1 / 2} \\
& =[n \operatorname{VaR}(X)]^{1 / 2}=n^{1 / 2} \operatorname{VaR}(X)
\end{aligned}
$$

lo cual es posible dado que las reclamaciones son independientes entre si, entonces el $V$ a R de la cartera de seguros como una distribución Poisson compuesta 
se puede escribir como

$$
\begin{aligned}
\operatorname{VaR}\left(S_{T}\right) & =\mathrm{P}_{\mathrm{r}}(N=0)+\sum_{j=1}^{\infty}\left[j V a R^{2}(X)\right]^{1 / 2} \mathrm{P}_{\mathrm{r}}(N=j) \\
& =e^{-\lambda}+\sum_{j=1}^{\infty}\left[j^{1 / 2} \operatorname{VaR}(X)\right] \frac{e^{\lambda} \lambda^{n}}{j !} \\
& =e^{-\lambda}+\sum_{j=1}^{\infty} \sqrt{j}\left[u+\frac{\hat{\beta}}{\hat{\xi}}\left\{\left(\frac{n}{N_{u}}(1-q)\right)^{-\xi}-1\right\}\right] \frac{e^{-\lambda} \lambda^{n}}{j !},
\end{aligned}
$$

Por otro lado, la estimación de la pérdida esperada condicional de la cartera de seguros puede expresarse como

$$
\begin{aligned}
\operatorname{PEC}\left(S_{T}\right) & =\mathrm{P}_{\mathrm{r}}(N=0)+\sum_{j=1}^{\infty}\left[\sum_{i=1}^{n} P E C^{2}\left(X_{i}\right)\right]^{1 / 2} \operatorname{Pr}_{\mathrm{r}}(N=j) \\
& =e^{-\lambda}+\sum_{j=1}^{\infty} \sqrt{j}\left[\frac{V a R_{q}}{1-\xi}+\frac{\beta+\xi u}{1-\xi}\right] \frac{e^{-\lambda} \lambda^{n}}{j !}
\end{aligned}
$$

expresión que permite el cálculo de la pérdida esperada condicional para diferentes niveles de confianza.

Calculando el cociente de la pérdida esperada condicional y el Valor en Riesgo así definidos, de las relaciones entre las ecuaciones se puede deducir que el cociente tiende hacia el valor de $1 /(1-\xi)$ cuando el nivel de confianza aumenta (o $q$ tiende a cero).

\section{Conclusiones}

En este trabajo se han utilizado los modelos de eventos extremos para construir las definiciones del Valor en Riesgo y la. Pérdida Esperada Condicional que permiten la modelación de procesos de colas pesadas, tales como son los rendimientos de los instrumentos financieros y las reclamaciones de los usuarios en una cartera de seguros.

Los modelos de eventos extremos ofrecen valores más altos para el $V a R$, pues el mayor peso de las colas de la distribución de probabilidad de los eventos estudiados implican que habrá una frecuencia mayor que la asumida bajo una ley de probabilidad normal. La pérdida esperada condicional también ofrece un resultado mayor que el de una distribución normal y bajo el modelo de eventos extremos queda totalmente determinada por el $V a R$ y los parámetros de la distribución Pareto Generalizada, que es la distribución que modela a las colas de los eventos.

Empíricamente en este trabajo se muestra que los portafolios de inversión utilizados por una aseguradora se caracterizan por tener rendimientos de colas pesadas, así como las reclamaciones de siniestros de un tipo de operaciones de salud. 
También se ha propuesto el $V a R$ de un portafolio para estimar el $V a R$ de una cartera de seguros, en donde además el monto total de reclamaciones se expresa como una variable aleatoria que sigue un proceso Poisson compuesto. Estos resultados son también aplicables para la pérdida esperada condicional.

\section{Bibliografía}

Artzner, P., F. Delbaen, J. Eber, and D. Heath (1997). Thinking Coherently, Risk, 10(11), pp. 68-71.

Artzner, P., F. Delbaen, J. Eber, and D. Heath (1999). Coherent Measures of Risk. Mathematical Finance, 9, pp. 203-228.

Acerbi, C. and D. Tasche (2001). On the Coherence of Expected Shortfall. ETH preprint.

Aguilera, M. (2002). Perspectivas para el desarrollo del sector asegurador. CNSF, Ponencia presentada en la XII Convención Nacional de Aseguradores.

Aguilera, M. (2003). Cambio estructural y desarrollo del sector asegurador mexicano. CNSF, Ponencia presentada en la XIII Convención Nacional de Aseguradores.

Bollerslev, T. (1986). Generalized Autoregressive Conditional Heteroscedasticity. Journal of Econometrics, 31, pp. 307-327.

Diario Oficial de la Federación. Reglas para la inversión de reservas técnicas de las instituciones y sociedades mutualistas de seguros. 18 de agosto de 2000.

Diario Oficial de la Federación. Acuerdo por el que se modifican la novena y décima quinta Reglas para la inversión de reservas técnicas de las instituciones y sociedades mutualistas de seguros. 13 de agosto de 2001.

Diario Oficial de la Federación. Acuerdo por el que se modifican la décima sexta de las Reglas para la inversión de reservas técnicas de las instituciones y sociedades mutualistas de seguros. 6 de febrero de 2003.

Embrechts, P. (2000). Extreme value theory: Potential and limitations as an integrated risk management tool. Working paper, ETH, Zurich.

Embrechts, P., S. Resnick, and G. Samorodnitsky (1998). Living on the edge. it RISK, 11(1), pp. 96-100.

Emmer, S., C. Klüpperlberg, and M. Trüsdedt (1998). VaR - a measure for the extreme risk. Solutions, 2, pp. 53-63.

Engle, R. (1982). Autoregressive Conditional Heteroscedasticity with estimates of the variance of United Kingdom inflations. Econometrica, 50, pp. 987-1008.

Engle, R., D. Lilien, and R. Robins (1987). Estimating Time Varying Risk Premia in the Term Structure: The ARCH-M Model. Econometrica, 55, pp. 391-407.

Jarrow, R. and E. Roselfeld (1984). Jump Risks and the Intertemporal Capital Asset Pricing Model. Journal of Business, 57, pp. 337-351.

Jorion, P. (1997). Value at risk: the new benchmark for controlling market risk. Chicago: Irwin Professional Pub.

Klüpperlberg; C.(2000). Developments in Insurance Mathematics. ETH preprint.

McNeil, A. (1999). Extreme Value Theory for Risk Managers. Internal Modelling and CAD II, RISK Books, pp. 93-113.

McNeil, A. and R. Frey (2000). Estimation of Tai-related Risk Measures for Heteroscedastic Financial Time Series: An Extreme Value Approach. Journal of Empirical finance, 7(3-4), pp. 271-300.

Nelson, D. (1991). Conditional Heteroskedasticity in Asset Returns: A New Approach. Econometrica, 59, pp. 347-370.

Pickands, J. (1975). Statistical Inference using Extreme Order Statistics. The Annals of Statistics, 3, pp. 15-102.

Smith, R. (2002). Statistics of Extremes with Applications in Environment, Insurance and Finance. Disponible en Internet: www.stat.unc.edu

Tajvidi, N. (1996). Multivariate Generalized Pareto Distributions. ETH preprint.

Venegas, F. (2001). Opciones, cobertura y procesos de difusión con saltos: una aplicación de los títulos de GCARSO. Estudios Económicos, 16 (2). 